Supplement of Web Ecol., 16, 9-12, 2016

http://www.web-ecol.net/16/9/2016/

doi:10.5194/we-16-9-2016-supplement

(C) Author(s) 2016. CC Attribution 3.0 License.

(c) (i)

Supplement of

\title{
Protected areas network and conservation efforts concerning threatened amphibians in the Brazilian Atlantic Forest
}

\section{F. S. Campos et al.}

Correspondence to: F. S. Campos (feliperoots@hotmail.com)

The copyright of individual parts of the supplement might differ from the CC-BY 3.0 licence. 


\section{Supplementary Table}

Table S1. Threatened amphibian species from the Brazilian Atlantic Forest according to their National Red List categories (MMA, 2014). CR = Critically Endangered, EN = Endangered, $\mathrm{VU}=$ Vulnerable.

\begin{tabular}{|c|c|c|}
\hline Family & Species & Category \\
\hline Aromobatidae & Allobates olfersioides & VU \\
\hline Brachycephalidae & Brachycephalus pernix & CR \\
\hline Brachycephalidae & Ischnocnema manezinho & VU \\
\hline Bufonidae & Melanophryniscus admirabilis & $\mathrm{CR}$ \\
\hline Bufonidae & Melanophryniscus cambaraensis & VU \\
\hline Bufonidae & Melanophryniscus dorsalis & VU \\
\hline Bufonidae & Melanophryniscus macrogranulosus & EN \\
\hline Bufonidae & Melanophryniscus setiba & $\mathrm{CR}$ \\
\hline Craugastoridae & Holoaden bradei & CR \\
\hline Craugastoridae & Holoaden luederwaldti & EN \\
\hline Cycloramphidae & Cycloramphus diringshofeni & CR \\
\hline Cycloramphidae & Cycloramphus faustoi & CR \\
\hline Cycloramphidae & Cycloramphus ohausi & EN \\
\hline Cycloramphidae & Thoropa petropolitana & EN \\
\hline Cycloramphidae & Thoropa saxatilis & VU \\
\hline Eleutherodactylidae & Adelophryne maranguapensis & VU \\
\hline Hylidae & Agalychnis granulosa & VU \\
\hline Hylidae & Aparasphenodon pomba & $\mathrm{CR}$ \\
\hline
\end{tabular}




\begin{tabular}{|c|c|c|}
\hline Hylidae & Bokermannohyla vulcaniae & $\mathrm{CR}$ \\
\hline Hylidae & Hypsiboas curupi & VU \\
\hline Hylidae & Hypsiboas cymbalum & $\mathrm{CR}$ \\
\hline Hylidae & Hypsiboas semiguttatus & EN \\
\hline Hylidae & Phyllodytes gyrinaethes & $\mathrm{CR}$ \\
\hline Hylidae & Scinax alcatraz & $\mathrm{CR}$ \\
\hline Hylidae & Scinax duartei & VU \\
\hline Hylidae & Scinax faivovichi & VU \\
\hline Hylidae & Scinax peixotoi & $\mathrm{CR}$ \\
\hline Hylidae & Xenohyla truncata & EN \\
\hline Hylodidae & Crossodactylus dantei & EN \\
\hline Hylodidae & Crossodactylus lutzorum & $\mathrm{CR}$ \\
\hline Leptodactylidae & Physalaemus caete & EN \\
\hline Leptodactylidae & Physalaemus maximus & VU \\
\hline Leptodactylidae & Physalaemus soaresi & $\mathrm{CR}$ \\
\hline Leptodactylidae & Paratelmatobius lutzii & $\mathrm{CR}$ \\
\hline Microhylidae & Chiasmocleis alagoanus & EN \\
\hline Odontophrynidae & Proceratophrys moratoi & EN \\
\hline Odontophrynidae & Proceratophrys palustris & $\mathrm{CR}$ \\
\hline Odontophrynidae & Proceratophrys sanctaritae & $\mathrm{CR}$ \\
\hline
\end{tabular}

